\title{
Prematuridade e outros fatores de risco adicional ao desenvolvimento perceptomotor e sua influência no desempenho escolar
}

\section{Prematuritand additional risk factors to perceptomotor development and it's influence on school performance}

\author{
Prematuridad y otros factores de riesgo adicional al desarrollo \\ perceptomotor y su influencia en el rendimiento escolar
}

\section{Débora Morais Pereira*}

Centro de Reabilitação Lucy Montoro, São José dos Campos, São Paulo, Brasil

\section{Rita de Cássia Tibério Araújo**}

Universidade Estadual Paulista Júlio de Mesquita Filho, UNESP, Marília, São Paulo, Brasil

\section{Ligia Maria Presumido Braccialli***}

Universidade Estadual Paulista Júlio de Mesquita Filho, UNESP, Marília, São Paulo, Brasil

\begin{abstract}
RESUMO
Considerando que múltiplos fatores podem afetar o desempenho de alunos em tarefas acadêmicas, foram objetivos deste estudo: a) avaliar o desempenho de alunos do Ensino Fundamental sob parâmetros da performance em testes padronizados; b) identificar a influência da ocorrência de nascimento pré-termo e/ou com baixo peso e da presença de deficiência sobre as habilidades avaliadas nos testes. Participaram 77 alunos do $2^{\circ}$ ano de uma escola pública. Sete deles nasceram prematuros e/ou com baixo peso e três foram apontados por seus responsáveis como apresentando deficiência. Para a coleta de dados foram utilizados o Teste do Desenvolvimento da Integração Visuo-Motora (Beery VMI) e o Teste de Desempenho Escolar (TDE) e um questionário desenvolvido para caracterização dos participantes. Em relação ao desempenho dos alunos no Beery VMI as médias de pontuações encontradas foram: 18 na avaliação da integração visuo-motora, 21 na avaliação de percepção visual e 22 no teste de coordenação motora. Em relação ao desempenho no TDE, a média de acertos foi: 13 no subteste de escrita, 8 na avaliação de aritmética e 46 no subteste de leitura. Constatou-se correlação positiva entre a habilidade de integração visuo-motora e a habilidade acadêmica avaliada nas tarefas de escrita, aritmética e leitura $(p \leq 0,05)$. A presença de deficiência e a ocorrência de prematuridade ou baixo peso ao nascimento acentuaram as correlações.
\end{abstract}

Palavras-chave: educação, fatores de risco, desempenho psicomotor.

n. 2 


\begin{abstract}
Considering that multiple factors can affect student performance on academic tasks, the -objectives of this study were: a) to evaluate the elementary student's performance in standardized tests, b) to identify the influence of preterm birth and/or low birth weight occurrence and the presence of disability on skills performance assessed in the tests. The participants were 77 students attending 2 nd grade of a elementary public school. Seven were born prematurely and / or underweight and three were appointed by those responsible as having a disability. For data collection the Developmental Test of Visual-Motor Integration (Beery VMI) and Teste de Desempenho Escolar (TDE) and a questionnaire developed for participants' characterization were used. Regarding the students' performance in Beery VMI the average scores were: 18 in visual-motor integration test, 21 in visual perception test and 22 in motor coordination test. Regarding the performance in TDE, the mean score was 13 in writing subtest, 8 in arithmetic subtest and 46 in reading subtest.A positive correlation between the visual-motor integration ability and writing, math and reading academic skills . $p \leq 0.05)$ was found. The presence of disability and the preterm birth and/or low birth weight occurrence accentuated the correlations.
\end{abstract}

Keywords: education, risk factors, psychomotor performance.

\begin{abstract}
RESUMEN
Mientras que varios factores pueden afectar al rendimiento de estudiantes em tareas acadêmicas, fueron objetivos de este estúdio: a) evaluar el desempeño de estudiantes en escuela primaria bajo parámetros de rendimiento en pruebas estandarizadas, b) identificar la influencia de ocurrencia de parto prematuro y/o de bajo peso al nacer y la presencia de discapacidad en habilidades evaluadas en las pruebas. Participaran 77 estudiantes de $2^{\circ}$ curso de una escuela pública. Siete prematuros y/o de bajo peso y tres presentavam discapacidad. Para la recolección de datos se utilizaron el Developmental Test of Visual-Motor Integration, el Teste de Desempenho Escolar y un cuestionario desarrollado para la caracterización de los participantes. En cuanto al rendimiento de los estudiantes en el Beery VMI fueron encontrado las siguiente puntuaciones medias: 18 en la evaluación de la integración visual-motor, 21 en la evaluación de la percepción visual y 22 en la prueba de la coordinación motora. En cuanto a la TDE rendimiento, la puntuación media fue de 13 por escrito substeste , 8 en la evaluación de la aritmética y 46 en lectura subprueba. Se encontró una correlación positiva entre la capacidad de integración visuo-motor y las tareas académicas de escritura, aritmética y lectura $(p \leq 0,05)$. La presencia de discapacidad y la incidencia de prematuridad y/o bajo peso al nacer hizo las correlaciones más acentuado.
\end{abstract}

Palabra clave: educación, factores de riesgo, rendimiento psicomotor.

\title{
1 Introdução
}

O processo de aprender a interagir com o ambiente inicia-se a partir do nascimento, sendo essa interação um processo tão perceptivo quanto motor (Gallahue \& Ozmun, 2005). Exige interação entre o cérebro, o corpo e o meio ambiente e envolve a memória de experiências passadas, a motivação, as expectativas, a seleção de 
informação sensorial e a busca ativa de informação sensorial pertinente (Lundy-Ekman, 2004; Haywood \& Getchell, 2010).

O processamento de informação proveniente do ambiente, pelo indivíduo, depende de funções neuropsicológicas de atenção, percepção e memória que sustentam a aprendizagem, entre outros fatores (Dias, 2004).

O desenvolvimento de habilidades perceptivas é dependente tanto da experiência como da maturação. A maturação desempenha papel importante no desenvolvimento da crescente precisão da percepção, porém, grande parte da melhoria na precisão deve-se à experiência (Gallahue \& Ozmun, 2005).

Pesquisas indicam que, à medida que as crianças passam pelos estágios de desenvolvimento motor, suas habilidades perceptivas tornam-se mais acentuadas e refinadas, o que se deve à crescente complexidade do aparato neuromuscular e dos receptores sensoriais e à crescente habilidade das crianças para explorar e movimentar-se pelo ambiente (Gallahue \& Ozmun, 2005).

As considerações sobre a percepção e a motricidade levaram à Psicologia o desenvolvimento de duas correntes de pensamento: de um lado os pesquisadores que firmam a tese de que elas são habilidades distintas e autônomas, e, do outro, aqueles que acreditam que ocorre um processo de interação entre elas (Pinelli Júnior, 1990).

Desse aparato teórico, surgiram vários instrumentos de avaliação, em que uma parte deles procura avaliar a percepção visual; e outra mede as habilidades motoras separadamente, existindo ainda aqueles calcados na tese da integração entre ambas as habilidades.

Existem diferentes nomenclaturas que se referem à interação entre a informação visual percebida e a execução coordenada de movimentos. Para Rosa Neto (2002), a coordenação visuo-motora é um processo de ação em que existe coincidência entre o ato motor e a estimulação visual percebida. Para Beery e Beery (2010) a integração visuo-motora é o nível em que a percepção visual e os movimentos dos dedos da mão são bem coordenados. A coordenação visuomanual é, para Rosa Neto (2002) e Sanghavi e Kelkar (2005), uma habilidade de interação harmoniosa entre os olhos e as mãos, envolvendo a percepção visual e a coordenação olho-mão, elaborada de modo progressivo com a evolução motora da criança e do aprendizado e que exige a participação de diferentes centros nervosos motores e sensoriais (Rosa Neto, 2002; Sanghavi \& Kelkar, 2005). Segundo Lustosa, Fiorentin e Rocha (2004), a coordenação óculo-manual busca a harmonia do movimento e a obtenção dos resultados almejados, relacionando-se com o controle do olhar que acompanha a mão enquanto a mesma desempenha alguma função. Neste estudo utilizaremos a terminologia e definição de Beery e Beery (2010) para integração visuo-motora. 
A relação entre as dificuldades escolares e os aspectos perceptuais e motores, em especial a integração visuo-motora, vem sendo discutida com frequência na literatura. A aprendizagem da leitura e da escrita é um processo complexo e difícil para as crianças em fase inicial de alfabetização (Trevisan, Coppede, \& Capellini, 2008). Envolve habilidades cognitivas, lingüísticas e motoras, as quais exigem o uso de componentes sensório-motores e perceptivos para decodificação das palavras e ação adequada no ato da escrita (Capellini \& Souza, 2008).

Problemas no controle motor fino e/ ou baixa habilidade perceptiva podem prejudicar o desempenho em tarefas de leitura, escrita e aritmética, afetando o aprendizado da criança na sala de aula, suas conquistas, motivações e autoestima (McHale \& Cermark, 1992; Beery \& Beery, 2010).

A importância de se olhar de maneira mais cuidadosa para aquelas crianças que apresentam fatores considerados como de risco para o desenvolvimento de habilidades percepto-motoras, entre elas, as crianças com histórico de prematuridade também vêm sendo destacada na literatura (Carvalho \& Magalhães, 2004).

De acordo com o Ministério da Saúde, apesar da prematuridade e baixo peso ao nascer não serem os únicos critérios que classificam o neonato como recém-nascido de risco, são os mais relevantes, sendo que, quanto menor o peso de nascimento e a idade gestacional maior o risco de comprometimento do desenvolvimento neurológico (Pessoa, Martins, Lima, \& Gaíva, 2015).

Teoricamente, qualquer recém-nascido pré-termo pode cursar com problemas futuros, mas há evidências de maior vulnerabilidade a morbimortalidade para aqueles nascidos com menos de 32 semanas e com peso inferior a $1500 \mathrm{~g}$ (Braga \& Sena, 2013).

Muitas são as evidências de que crianças pré-termo estão sob maior risco para apresentar atraso perceptual, motor e cognitivo, associado ou não a problemas de comportamento e déficit de atenção (Magalhães, Catarina, Barbosa, Mancini, \& Paixão, 2003).

Os bebês prematuros podem apresentar alta incidência de limitações no seu desenvolvimento, resultando em um aumento do uso de serviços terapêuticos e educacionais especiais em idade escolar (Formiga \& Linhares, 2009).

Entre os problemas do desenvolvimento apresentados pela criança prematura, as alterações severas do desenvolvimento motor são de mais fácil identificação e geralmente podem ser detectadas no primeiro ano de vida, no entanto, alterações discretas na coordenação motora podem passar despercebidas (Magalhães, Rezende, Magalhães, \& Albuquerque, 2009).

Por outro lado, a prematuridade e o baixo peso ao nascer não são as únicas variáveis que parecem influenciar no desenvolvimento e desempenho em atividades escolares. 
Atrasos no desenvolvimento são produzidos pela combinação de fatores de risco genético, biológico, psicológicos e ambientais, envolvendo interações complexas entre elas (Rodrigues \& BolsoniSilva, 2011). As dificuldades escolares também estão relacionadas a fatores múltiplos, tais como nível cognitivo, nível social, comportamento, nível de desenvolvimento da linguagem, método de ensino, habilidades motoras e habilidades de organização perceptual (Oliveira, Lopes, \& Magalhães, 2004).

Levando em conta os múltiplos fatores que podem afetar o desempenho de alunos em tarefas acadêmicas, foram objetivos deste estudo: a) avaliar a habilidade de alunos ingressantes no Ensino Fundamental sob parâmetros da performance no Teste do Desenvolvimento da Integração Visuo-Motora (Beery VMI) e no Teste de Desempenho Escolar (TDE); b) identificar a influência da ocorrência de nascimento pré-termo e/ou com baixo peso e de outros fatores sugestivos de risco de alteração da habilidade avaliadas nos testes.

\section{Método}

\subsection{Participantes}

Participaram do estudo 77 alunos do $2^{\circ}$ ano do ensino fundamental de uma escola pública do interior paulista, sendo 40 meninas e 37 meninos. A idade das crianças variou de 6 anos e 10 meses a 8 anos e 7 meses, com média de 7 anos e 6 meses.

Das 77 crianças avaliadas, três foram apontadas pelos responsáveis, mediante preenchimento de questionário sobre dados da criança, como apresentando alguma deficiência. Segundo um dos responsáveis, uma das crianças realizou cirurgia devido a glaucoma, perdendo $85 \%$ da visão do olho direito e $15 \%$ da visão do olho esquerdo, sendo usuário de óculos. No questionário das duas outras crianças não havia informações que especificavam o tipo de deficiência. Segundo relato das professoras, uma das crianças apresentava déficit auditivo sem uso de aparelho auditivo e a outra apresentava déficit cognitivo. Contudo, não foram apontados outros indicativos sugestivos de prejuízo na realização das atividades de nenhum dos três alunos, não havendo também menção a qualquer tipo de auxílio na realização das atividades acadêmicas.

Os participantes do estudo também foram classificados segundo a idade gestacional e o peso ao nascimento. Do total das crianças avaliadas neste estudo, 70 nasceram com idade gestacional $\geq 37$ semanas e peso $\geq 2500 \mathrm{~g}$ e as outras sete crianças nasceram prematuras ( $<37$ semanas) e/ou com baixo peso $(<2500 \mathrm{~g})$. 


\subsection{I nstrumentos}

Para a coleta de dados foram utilizados os instrumentos Developmental Test of Visual-Motor Integration - Beery VMI (Teste do Desenvolvimento da Integração Visuo-Motora), o Teste de Desempenho Escolar (TDE) e um questionário desenvolvido para caracterização dos alunos quanto a condições do nascimento (idade gestacional, peso e estatura ao nascimento) e presença de deficiência.

O Beery VMI é um teste utilizado para avaliar a habilidade de integração visuo-motora. Esse instrumento possui dois testes suplementares, quais sejam o Teste de Percepção Visual e o Teste de Coordenação Motora. No teste de integração visuo-motora é solicitada a reprodução, em um espaço específico, de 24 figuras geométricas apresentadas. No teste de percepção visual, as mesmas figuras são disponibilizadas na mesma ordem, mas em tamanho menor e mais próximas, e o avaliando deve identificar entre figuras semelhantes, aquela que é exatamente igual à apresentada em local destacado. No teste de coordenação motora o indivíduo que está sendo avaliado deve ligar os pontos que estão dentro das figuras geométricas sem ultrapassar o espaço determinado. Em todos os testes do Beery VMI a pontuação máxima possível (Escore Bruto) é de 30 pontos (Beery \& Beery, 2010).

Não há até o momento estudos que realizaram a normatização deste instrumento para amostras brasileiras, no entanto, Pinelli Júnior (1990) indicou em sua dissertação de mestrado que o Beery VMI se mostrou adequado e válido como medida psicométrica numa amostra brasileira.

Apesar de não haver até o momento estudos que realizaram a normatização deste instrumento para amostras brasileiras, essa situação não se configura como um obstáculo metodológico neste estudo, pois não foi objetivo deste trabalho verificar se a pontuação obtida pelos escolares estava dentro do esperado para sua faixa etária. Em nossa pesquisa, os resultados obtidos são discutidos com base nos escores obtidos em outro estudo nacional.

O TDE é um instrumento psicométrico, desenvolvido para a população brasileira, que busca oferecer, de forma objetiva, uma avaliação das capacidades fundamentais para o desempenho escolar. É composto por três subtestes, sendo eles o de escrita, o de aritmética e o de leitura. O subteste de escrita consiste na escrita do próprio nome e de palavras isoladas apresentadas sob a forma de ditado; o subteste de aritmética exige a solução oral de problemas e cálculos de operações aritméticas por escrito; o subteste de leitura é realizado por meio do reconhecimento de palavras isoladas do contexto. Cada um dos subtestes apresenta uma escala de itens em ordem crescente de dificuldade (Stein, 1994). 
O questionário para caracterização dos alunos foi entregue, preenchido e devolvido no mesmo dia. Foi necessário realizar contato telefônico com três responsáveis para a complementação de informações de peso ao nascimento, pois esses não souberam informar esses dados no dia da reunião. As informações sobre idade gestacional, peso, estatura ao nascimento foram referidas pelos responsáveis sem confirmação pela pesquisadora em carteira de saúde da criança e, no caso de indicação de deficiência, foram coletadas informações adicionais fornecidas pelas professoras.

\subsection{Considerações Éticas}

O manuscrito em questão é inédito, não foi financiado e é resultado de dissertação de mestrado. Foi encaminhado ao Comitê de Ética em Pesquisa e aprovado.

Os esclarecimentos quanto ao projeto foram fornecidos aos responsáveis pelas crianças durante a reunião de pais e professores, no início do $4^{\circ}$ bimestre do ano letivo. Todos aqueles que concordaram com a participação da criança neste estudo assinaram a concordância com o Termo de Consentimento Livre e Esclarecido (TCLE), atendendo à Resolução 196/96.

Levando em conta o compromisso assumido com a escola e com os responsáveis pelos participantes, após a análise geral dos dados coletados, os resultados foram apresentados aos professores, tendo sido redigido e disponibilizado aos responsáveis um relatório com o desempenho obtido pelas crianças nos testes aplicados. No caso dos alunos que apresentaram atraso importante na habilidade de integração visuo-motora, de percepção visual e/ou coordenação motora foram fornecidas, à escola e aos responsáveis, sugestões de atividades para auxiliar no desenvolvimento destas habilidades, baseadas no programa de treinamento da percepção visual, coordenação motora e integração visuo-motora, proposto por Zafani e Araújo (2010).

\subsection{Coleta de Dados}

A aplicação dos instrumentos de coleta de dados ocorreu durante o horário escolar, mas em sala reservada especificamente para este fim. Inicialmente foi aplicado o teste de integração visuo-motora, coletivamente em grupos de cinco ou seis alunos. O TDE e os testes suplementares do Beery VMI foram aplicados individualmente. A aplicação dos testes ocorreu em dias diferentes para não cansar os alunos, e a ordem da aplicação dos testes (Beery VMI e TDE) variou entre os participantes. A coleta de dados foi realizada diariamente, conforme disponibilidade dos professores, durante um período de aproximadamente dois meses. 
Quanto aos alunos com informações sugestivas de deficiência, alguns cuidados foram tomados. A versão do TDE utilizada pela criança com relato de déficit visual foi ampliada para melhor visualização dos números no subteste de matemática e das palavras no subteste de leitura. No subteste de escrita foi deixado um espaço maior para a criança escrever as palavras ditadas. Considerando que as figuras apresentadas pelo teste de integração visuo-motora são relativamente grandes, e que a modificação no teste de percepção visual e coordenação motora poderiam comprometer o resultado, não foram realizadas alterações no Beery VMI.

$\mathrm{Na}$ aplicação dos testes com a criança com relato de déficit auditivo foi tomado o cuidado de articular bem a fala, principalmente no ditado das palavras. Não foram realizadas alterações estruturais nos testes.

Por fim, para aplicar o teste na criança com relato de déficit intelectual, não foram realizadas alterações estruturais nos testes, no entanto, a aplicação do Beery VMI foi iniciada na figura 4. Como a criança foi capaz de pontuar nos itens de 4 a 7 (imitação das figuras), foi dado prosseguimento ao teste de maneira semelhante à aplicação com as demais crianças.

\subsection{Análise dos dados}

A pontuação dos testes aplicados foi realizada conforme instruções contidas em seus manuais. Para medir o grau de correlação entre o desempenho dos alunos no Beery VMI e no TDE foi utilizado o coeficiente de correlação de Pearson, adotando o nível de significância de $5 \%(\leq 0,05)$.

Uma primeira análise dos resultados foi realizada levando-se em consideração o desempenho de todas as crianças $(\mathrm{N}=77)$. Outra análise foi realizada separando-se as crianças com informações sugestivas de deficiência e as crianças que nasceram pré-termo $(<37$ semanas) e/ou com baixo peso $(<2500 \mathrm{~g})$.

\section{Resultados}

\subsection{Desempenho dos alunos nos testes aplicados}

Em relação ao desempenho dos alunos no Beery VMI, a pontuação na avaliação da integração visuo-motora variou de 10 a 25, sendo a média de 18 acertos. Na avaliação da percepção visual os alunos tiveram uma pontuação média de 21 , sendo 7 a menor pontuação e 27 a maior. E no teste de coordenação motora, a pontuação variou de 15 a 28 , sendo 22 a média alcançada. 
Em relação ao desempenho no TDE, a média de acertos no subteste de escrita foi 13 , sendo a variação de zero a 28 acertos. Na avaliação de aritmética, a pontuação variou de zero a 14, sendo a média de 8 pontos. E no subteste de leitura, houve uma variação de zero a 70, sendo a média de 46 pontos.

No manual para aplicação e interpretação do TDE (Stein, 1994) são fornecidos parâmetros para classificação de escores brutos de acordo com a série escolar. Ao realizar a conversão do Escore Bruto das crianças avaliadas neste estudo, segundo os parâmetros disponibilizados pelo manual para alunos da antiga $1^{\text {a }}$ série do Ensino Fundamental (atual $2^{\circ}$ ano), obteve-se 0 resultado que pode ser visualizado na Figura 1.

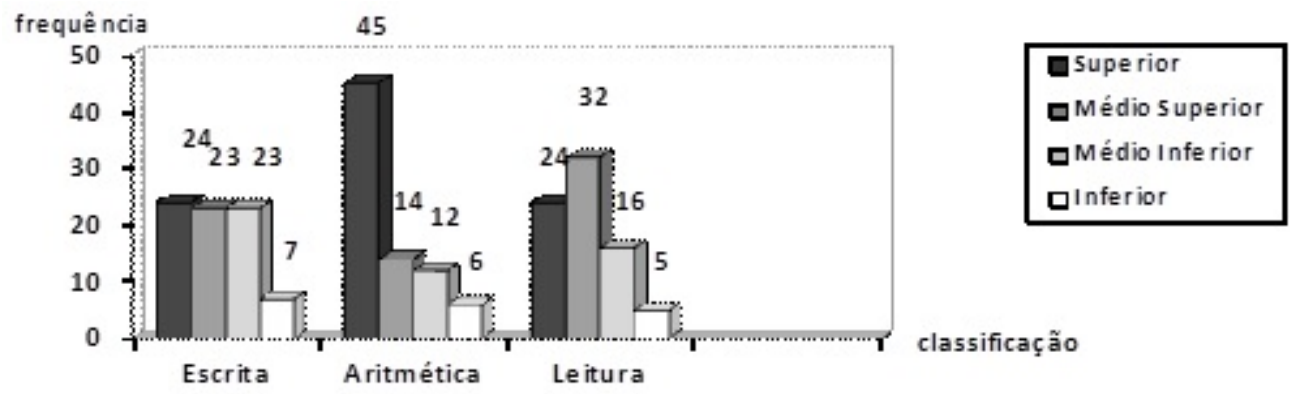

Figura 1 - Distribuição da classificação do desempenho dos alunos no Teste de Desempenho Escolar (subtestes de escrita, aritmética e leitura) segundo a Tabela de Normas para alunos da $1^{\text {a }}$ série do Ensino Fundamental.

Somando a frequência de classificação do desempenho superior e médio superior de cada um dos subtestes do TDE e transformando esse número em porcentagem encontra-se que $61 \%$ das crianças avaliadas apresentaram desempenho superior e médio superior em escrita; $76 \%$ apresentaram o mesmo desempenho em aritmética e $72 \%$ em leitura. Observa-se, portanto, neste estudo, um desempenho superior ao previsto para a série escolar.

A Tabela 1 permite a análise em separado do desempenho das crianças com risco adicional (crianças com informações sugestivas de deficiência e crianças que nasceram prematuras e/ou com baixo peso), segundo a tabela de normas para alunos da $1^{a}$ série do Ensino Fundamental. 
Tabela 1

Classificação do desempenho das crianças com risco adicional segundo a tabela de normas do Teste de Desempenho Escolar para alunos da $1^{\text {a }}$ série do Ensino Fundamental.

\begin{tabular}{|c|c|c|c|}
\hline \multirow{2}{*}{ CONDIÇÃO DE RISCO } & \multicolumn{3}{|c|}{ CLASSIFICAÇÃO } \\
\hline & Escrita & Aritmética & Leitura \\
\hline $\begin{array}{l}\text { deficiência intelectual } \\
\text { deficiência auditiva } \\
\text { deficiência visual }\end{array}$ & $\begin{array}{c}\text { Inferior } \\
\text { médio inferior } \\
\text { médio superior }\end{array}$ & $\begin{array}{l}\text { inferior } \\
\text { médio inferior } \\
\text { superior }\end{array}$ & $\begin{array}{c}\text { Inferior } \\
\text { médio inferior } \\
\text { médio superior }\end{array}$ \\
\hline $\begin{array}{c}\text { prematuro e/ou baixo peso } \\
1\end{array}$ & médio inferior & superior & médio inferior \\
\hline $\begin{array}{c}\text { prematuro e/ou baixo peso } \\
2\end{array}$ & médio inferior & médio inferior & médio inferior \\
\hline$\underset{3}{\text { prematuro e/ou baixo peso }}$ & médio inferior & superior & médio inferior \\
\hline $\begin{array}{c}\text { prematuro e/ou baixo peso } \\
4\end{array}$ & médio superior & médio superior & médio superior \\
\hline prematuro e/ou baixo peso & superior & superior & médio superior \\
\hline $\begin{array}{c}\text { prematuro e/ou baixo peso } \\
6\end{array}$ & superior & superior & Superior \\
\hline $\begin{array}{c}\text { prematuro e/ou baixo peso } \\
7\end{array}$ & superior & superior & Superior \\
\hline
\end{tabular}

Analisando o desempenho das crianças com informações sugestivas de deficiência podemos observar que apenas a criança com indicação de déficit intelectual obteve classificação inferior segundo os parâmetros para a série em questão, e neste caso, o desempenho foi inferior nos três subtestes do TDE (escrita, aritmética e leitura). A criança com indicação de déficit auditivo apresentou desempenho classificado como médio inferior nos três subtestes. A criança com indicação de déficit visual apresentou pontuações classificadas como média superior e superior.

Dentre as crianças que nasceram prematuras e/ou com baixo peso não houve pontuação classificada como inferior segundo os parâmetros para a série escolar. A classificação no nível médio inferior foi obtida por três crianças nos subtestes de escrita e leitura e por uma criança no subteste de aritmética. As demais pontuações foram classificadas nos níveis médio superior e superior.

\subsection{Relação entre a habilidade de integração visuo-motora e o desempenho nas tarefas de escrita, aritmética e leitura}

Para verificar a correlação entre a habilidade de integração visuomotora avaliada pelo Beery VMI e as tarefas escolares de escrita, aritmética e leitura avaliadas pelo TDE foi aplicado o teste estatístico de correlação de Pearson. Os coeficientes de correlação de Pearson ( $r$ ) e o valor de " $p$ " obtidos podem ser verificados na Tabela 2, que reúne três situações de análises: amostra composta por todos os 
participantes $(\mathrm{N}=77)$, amostra composta pelas crianças sem indicação de fator de risco adicional (prematuridade, baixo peso e/ou presença de deficiências) $(\mathrm{N}=67)$, amostra composta somente pelas crianças com indicação de fator de risco adicional (prematuridade, baixo peso e/ou presença de deficiências) $(\mathrm{N}=10)$.

\section{Tabela 2}

Correlação de Pearson entre a pontuação no teste integração visuomotora e nas tarefas escolares de escrita, aritmética e leitura.

( $N=77$ : amostra composta por todos os participantes)

\begin{tabular}{|c|c|c|c|}
\hline Variáveis & & $\begin{array}{c}\text { Coeficiente de } \\
\text { correlaçáo de } \\
\text { Pearson (r) }\end{array}$ & $\begin{array}{c}\text { Valor de } \\
\text { P }\end{array}$ \\
\hline $\begin{array}{l}\text { Integração Visuo-Motora } \\
\text { Escrita }\end{array}$ & $x$ & 0,244 & 0,033 \\
\hline $\begin{array}{l}\text { Integração Visuo-Motora } \\
\text { Aritmética }\end{array}$ & $x$ & 0,277 & 0,015 \\
\hline $\begin{array}{l}\text { Integração Visuo-Motora } \\
\text { Leitura }\end{array}$ & $x$ & 0,230 & 0,044 \\
\hline \multicolumn{4}{|c|}{$\begin{array}{l}\text { ( } \mathrm{N}=67 \text { : amostra sem as crianças com indicação de fator de risco } \\
\text { adicional - prematuridade, baixo peso e/ou presença de deficiências) }\end{array}$} \\
\hline Variáveis & & $\begin{array}{c}\text { Coeficiente de } \\
\text { correlação de } \\
\text { Pearson (r) }\end{array}$ & $\begin{array}{c}\text { Valor de } \\
\text { "p" }\end{array}$ \\
\hline $\begin{array}{c}\text { Integração Visuo-Motora } \\
\text { Escrita }\end{array}$ & $x$ & 0,093 & 0,454 \\
\hline $\begin{array}{l}\text { Integração Visuo-Motora } \\
\text { Aritmética }\end{array}$ & $x$ & 0,164 & 0,184 \\
\hline $\begin{array}{l}\text { Integração Visuo-Motora } \\
\text { Leitura }\end{array}$ & $x$ & 0,092 & 0,461 \\
\hline
\end{tabular}

$(\mathrm{N}=10$ : amostra somente das crianças com indicação de fator de risco adicional - prematuridade, baixo peso e/ou presença de deficiências)

\begin{tabular}{cccc}
\hline Variáveis & & $\begin{array}{c}\text { Coeficiente de } \\
\text { correlação de } \\
\text { Pearson (r) }\end{array}$ & $\begin{array}{c}\text { Valor de } \\
\mathbf{p}\end{array}$ \\
\hline Integração Visuo-Motora & $\times$ & 0,807 & 0,005 \\
$\begin{array}{c}\text { Escrita } \\
\text { Integração Visuo-Motora } \\
\text { Aritmética }\end{array}$ & $\times$ & 0,686 & 0,029 \\
$\begin{array}{c}\text { Integração Visuo-Motora } \\
\text { Leitura }\end{array}$ & $\times$ & 0,733 & 0,016 \\
\hline
\end{tabular}

Os resultados obtidos no teste estatístico de correlação de Pearson (Tabela 2) indicaram a existência de uma correlação fraca $(r<0,5)$, no entanto significativa ( $p \leq 0,05)$, entre a habilidade de integração 
visuo-motora e as habilidades acadêmicas avaliadas para a amostra total $(\mathrm{N}=77)$.

A mesma análise estatística de correlação entre a habilidade de integração visuo-motora avaliada pelo Beery VMI e as tarefas de leitura, escrita e aritmética do TDE foi realizada retirando-se da amostra de 77 alunos as 7 crianças que nasceram prematuras e/ou com baixo peso e as 3 crianças caracterizadas pelos seus responsáveis como deficientes $(\mathrm{N}=67)$. Na análise dos dados da amostra $(N=67)$, cuja indicação de deficiência e ocorrência de prematuridade e baixo peso ao nascimento não foi referida pelos responsáveis, verifica-se que a correlação entre o desempenho no teste de integração visuo-motora e o desempenho nas tarefas de escrita, aritmética e leitura do TDE não foi estatisticamente comprovada (valor de $p>0,05$ ). Essa mesma análise estatística também foi realizada separadamente para os 10 alunos (crianças que nasceram prematuras e/ou com baixo peso e aquelas cujos responsáveis indicaram como deficientes). Observando o valor de " $p$ " e o coeficiente de correlação de Pearson obtidos nessa análise estatística $(\mathrm{N}=10)$, verifica-se que existiu uma correlação forte entre a habilidade de integração visuo-motora e a tarefa de escrita ( $r>$ $0,75)$; uma correlação média $(r>0,5)$ entre a pontuação obtida no teste de integração visuo-motora e a obtida nas tarefas de aritmética e uma correlação média $(r>0,5)$ entre a pontuação obtida no teste de integração visuo-motora e a obtida na leitura.

\section{Discussão}

O resultado obtido com aplicação do Teste de Desenvolvimento da Integração Visuo-Motora (Beery VMI) não pode ser comparado com a pontuação esperada para a idade disponibilizada pelo manual (Beery \& Beery, 2010), uma vez que este instrumento ainda não foi normatizado para amostras brasileiras.

No entanto, foi encontrado na literatura o estudo de Oliveira et al. (2004) que também estudaram a integração visuo-motora por meio da aplicação do Beery VMI e descreveram o desempenho no Beery VMI de 60 crianças com idade entre 6 e 7 anos. O resultado encontrado para as crianças de 7 anos foi um número médio de 16 acertos no teste de integração visuo-motora e de 18 acertos nos testes de percepção visual e de Coordenação Motora. Comparativamente com os resultados encontrados por essas autoras, em nosso estudo obtivemos médias superiores.

Diferentemente do Beery VMI, o Teste de Desempenho Escolar (TDE) foi desenvolvido para a população brasileira, fornecendo parâmetros para classificação do desempenho obtido nos subtestes de escrita, aritmética e leitura segundo a idade e série escolar. 
Analisando o desempenho dos alunos no TDE tendo sob parâmetro de comparação o desempenho esperado para a série escolar (antiga $1^{a}$ série do Ensino Fundamental) foram encontradas frequências elevadas de classificação superior e médio superior em todos os subtestes do TDE. A classificação da pontuação obtida pelas crianças com deficiência e pelas crianças que nasceram prematuras e/ou com baixo peso variou de maneira considerável.

Ao se aplicar o teste estatístico de correlação Pearson para investigar a relação entre a pontuação obtida no teste de integração visuomotora e a obtida nas tarefas escolares de escrita, aritmética e leitura para a amostra total $(\mathrm{N}=77)$, confirmou-se a hipótese de correlação entre as habilidades investigadas, no entanto é importante esclarecer que tal correlação foi fraca $(r>0,5)$.

A relação entre habilidades percepto-motoras, de um lado, e o desempenho em tarefas escolares, de outro lado, também vêm sendo objeto de pesquisa de outros autores. Kulp (1999) investigou a relação entre a habilidade de integração visuo-motora e o desempenho acadêmico. Foram incluídas nessa investigação 191 crianças do jardim de infância até a $3^{a}$ série do ensino fundamental de Cleveland, Ohio. Discriminação visual e espacial, coordenação motora fina visual e integração visuo-motora foram avaliadas com o Beery VMI. Os professores das salas de aula avaliaram os alunos em leitura, matemática e escrita em uma escala de 1 a 5 , sendo 1 a melhor performance e 5 representando a pior. Alunos do $2^{\circ}$ e $3^{\circ}$ ano também foram avaliados na capacidade de soletrar. O teste Standford Diagnostic Reading foi utilizado para avaliar a habilidade de leitura em todas as crianças e o teste Otis-Lennon School Ability (OLSAT) foi utilizado para avaliar a habilidade cognitiva dos alunos do $2^{\circ}$ ano. Os resultados da pesquisa mostraram que a performance no Beery VMI esteve significativamente relacionada com os resultados da avaliação do desempenho dos alunos em leitura, matemática, escrita e soletração, com a performance dos alunos das séries iniciais no Stanford Reading Test e com o desempenho dos alunos do $2^{\circ}$ ano no OLSAT. Gomes (1998) em sua dissertação de mestrado apresentou resultados que apontaram para a relação entre habilidades psicomotoras e o desempenho em tarefas escolares. Nesse estudo a autora focalizou o desempenho de alunos em atividade de escrita e aritmética, no intuito de entender quais fatores afetam o melhor e pior desempenho com destaque para duas variáveis: o nível de desenvolvimento cognitivo e o nível de desenvolvimento psicomotor. Participaram da pesquisa 146 crianças que freqüentavam as salas de $2^{a}$ e $3^{a}$ séries do primeiro grau de uma escola da rede Estadual de Ensino. Para selecionar a amostra foram aplicadas, coletivamente em sala de aula, provas escolares de aritmética e de escrita. A prova de aritmética foi constituída de 10 exercícios com atividades de sequência numérica, cálculos (soma, 
subtração, multiplicação e divisão) e problemas. A prova de escrita, por sua vez, foi composta pelo ditado de 3 textos, sendo a correção realizada segundo os erros em cada palavra. Os resultados dessas provas permitiram identificar os pólos: os alunos que apresentaram os menores índices de desempenho escolar, considerando a população estudada, e os que apresentaram o melhor desempenho. Aqueles com desempenho intermediário foram excluídos da pesquisa. A amostra foi composta por dois grupos, 30 alunos que apresentaram os menores índices de desempenho escolar e 30 alunos que apresentaram o melhor desempenho nas provas de escrita e aritmética. Dentre os 30 alunos de cada grupo, 15 deles eram da $2^{a}$ série, e os outros 15, da $3^{a}$ série. Para se avaliar os aspectos cognitivos, fez-se o uso de experimentos descritos por Piaget e Inhelder, e para avaliação da estrutura espacial foram utilizadas quatro modalidades da avaliação psicomotora: esquema corporal, lateralidade, estrutura espacial e estrutura temporal. Na comparação entre os dois grupos, de melhor e pior desempenho, de cada série, os resultados encontrados indicaram que a média de acertos nas avaliações de desenvolvimento cognitivo e no exame psicomotor foi maior entre as crianças que apresentaram melhores desempenhos em escrita e aritmética.

Ambrósio (2011) investigou a relação entre a psicomotricidade e a alfabetização dos alunos do $2^{\circ}$ ano. A autora partiu do pressuposto de que habilidades cognitivas, afetivas e motoras por parte da criança são necessárias para a aprendizagem e buscou verificar uma possível relação entre o desenvolvimento psicomotor de alunos do $2^{\circ}$ ano do ensino fundamental e o desempenho escolar no processo de alfabetização. Compuseram a pesquisa duas classes de $2^{\circ}$ ano do ensino fundamental da Rede Pública de Jundiaí, totalizando 40 alunos avaliados. Para a coleta de dados foram utilizados 5 instrumentos: Exame Psicomotor, Teste Gestáltico Visomotor de Bender B-SPG, Avaliação de Dificuldade na Aprendizagem da Escrita (ADAPE), Provinha Brasil e Teste de Inteligência Não-verbal - R2. O Exame Motor utilizado foi o proposto por Oliveira, no qual são realizadas diversas provas para observação do comportamento dos examinados segundo os seguintes aspectos: coordenação, equilíbrio, esquema corporal, imitação de atitudes, lateralidade, estruturação espacial e temporal e ritmo. Nesse estudo, a coleta de dados dividiu-se em duas etapas: a primeira ocorreu no início do ano, quando foram aplicados, de maneira individual, todos os instrumentos, com exceção da Provinha Brasil que foi aplicada pela escola no mês de maio e cujos resultados foram fornecidos pela administração escolar. Na segunda etapa, no final do ano, foram aplicados os mesmos instrumentos, e a Provinha Brasil foi reaplicada pela escola. Os resultados indicaram relação entre os resultados obtidos no exame psicomotor e no teste de Bender e entre os resultados da Provinha Brasil, do ADAPE e do 
teste de Bender. Não foi constatada tendência de associação dos resultados da Provinha Brasil com as habilidades psicomotoras. No entanto, foi observada relação entre as habilidades psicomotoras e a prova ADAPE. Segundo a autora, o estudo constatou associação entre habilidades psicomotoras, maturidade visuo-motora e desempenho escolar.

Suehiro e Santos (2005) também investigaram a relação entre tarefas escolares e habilidades perceptomotoras. Participaram deste estudo 287 crianças, de ambos os sexos, com idade entre 7 e 10 anos, das $2^{a}$ e $3^{a}$ séries de escolas públicas e particulares. Para a coleta de dados foi utilizado um questionário de identificação, a Escala de Avaliação de Dificuldades na Aprendizagem da Escrita (ADAPE) e o Teste Gestáltico Viso-Motor de Bender. Para verificar a hipótese de que os construtos medidos pelos instrumentos utilizados estavam relacionados, recorreu-se à prova de Correlação de Pearson. Os resultados da análise mostraram uma correlação de 0,434 $(p=0,000)$ entre os testes aplicados, indicando a existência de relação entre a aprendizagem da escrita e a capacidade perceptomotora.

Em nosso estudo, a correlação significativa evidenciada na amostra geral $(\mathrm{N}=77)$ entre a habilidade de integração visuo-motora avaliada pelo Beery VMI e as tarefas escolares de escrita, aritmética e leitura avaliadas pelo TDE dependeu do desempenho dos 10 alunos cujos responsáveis referiram prematuridade e/ou baixo peso ao nascimento e presença de deficiência.

A literatura científica tem apresentado alguns trabalhos que estudaram possíveis consequências do nascimento prematuro e/ou com baixo peso. Entre esses trabalhos temos a dissertação de Riechi (2008) e sua posterior publicação em periódico (Riechi, MouraRibeiro, \& Ciasca, 2011) que buscou verificar a associação entre o nascimento pré-termo e baixo peso e o desenvolvimento neuropsicológico de crianças e adolescentes, a fim de identificar diferenças no funcionamento cognitivo comportamental, assim como a prevalência do transtorno de aprendizagem em escolares nascidos pré-termo e com baixo peso. Para realizar esse estudo, a autora avaliou dois grupos de crianças: Grupo Propósito e Grupo Controle. O Grupo Propósito (GP) foi formado por 60 sujeitos em idade escolar que nasceram com idade gestacional inferior a 37 semanas e com peso $<2500 \mathrm{~g}$, e cuja idade variou entre 6 e 15 anos. O Grupo Controle (GC) foi constituído por 60 sujeitos irmãos ou vizinhos e colegas do escolar GP, nascidos a termo e com peso $\geq 2500 \mathrm{~g}$, sem intercorrência gestacional ou de parto e com idades equivalentes. Todos os participantes foram avaliados com os seguintes instrumentos: roteiro de anamnese, contendo dados da história do desenvolvimento físico, motor, social, cognitivo e comportamental da criança; questionário escolar, respondido pelo professor atual da 
criança, com 46 questões fechadas sobre o desempenho acadêmico e as habilidades cognitivo-comportamentais; questionário socioeconômico para caracterização do nível socioeconômico conforme critérios propostos pela Associação Brasileira de Institutos de Pesquisa de Mercado (ABIPEME); Escala Wechsler de Inteligência Infantil (WISC III); Teste Neuropsicológico Infantil Luria-Nebraska C; Teste de Desempenho Escolar (TDE); Figura Complexa de Rey; Teste Gestáltico Viso-Motor de Bender; Trail Making Test (TMT); Escala Comportamental A2 de Rutter; Lista de Verificação Comportamental para Crianças e Adolescentes; Exame Neurológico Tradicional (UNICAMP) e Exame Neurológico Evolutivo - ENE de Lefévre. Os resultados mostraram que os escolares que nasceram prematuros e com baixo peso apresentaram: a) diferenças significativas em funções neuropsicológicas específicas e na aprendizagem acadêmica; b) índices de QI menores, sendo a habilidade não-verbal de organização perceptual a maior defasagem intelectual observada; c) desempenho neuropsicológico inferior com comprometimentos específicos nas habilidades tátil-cinestésica, raciocínio matemático, viso-construtiva, memória visual e coordenação visuo-motora; d) desenvolvimento neurológico imaturo; e) pior desempenho escolar, com comprometimento nas atividades acadêmicas de aritmética e leitura; f) freqüência seis vezes maior de Distúrbios de Aprendizagem e frequência três vezes maior de Transtornos de Atenção.

Magalhães et al. (2003) também estudaram os possíveis efeitos da prematuridade ao buscar investigar a existência de diferenças significativas entre crianças, com o mesmo nível socioeconômico, nascidas pré-termo e a termo, em provas de equilíbrio, tônus postural e coordenação visuo-motora. Participaram deste estudo dois grupos de crianças. O Grupo I foi composto por 35 crianças nascidas até a $34^{a}$ semana de gestação e/ou com peso $\leq 1500 \mathrm{~g}$, entre 5 e 7 anos de idade, sem quadros severos diagnosticados como paralisia cerebral, retardo mental, entre outros e com renda familiar entre 1 e 3 salários mínimos. O Grupo II foi constituído de 35 crianças nascidas a termo e sem história de intercorrências neonatais, selecionadas por características de idade e sexo semelhantes às das crianças do Grupo I. Para a coleta de dados foram utilizados o Teste Gestáltico de Bender, o Teste de Acuidade Motora (Motor Accuracy Test - MAC) de Ayres, provas de equilíbrio estático e de tônus postural. Foram encontradas diferenças significativas de desempenho entre os dois grupos em todos os testes, apontando para um pior desempenho das crianças do Grupo I, exceto na avaliação de acuidade no teste MAC. Neste último teste foram encontradas diferenças significativas entre os grupos em relação ao tempo de execução do teste, tendo sido constatada uma maior lentidão na execução pelas crianças do Grupo I. Segundo as autoras, esse estudo dá suporte às evidências de que crianças com história de prematuridade apresentam pior desempenho 
em testes percepto-motores e ressaltam a importância do acompanhamento longitudinal dessas crianças, principalmente daquelas nascidas antes da $34^{a}$ semana de gestação com peso $\leq$ $1500 \mathrm{~g}$.

\section{Conclusão}

De uma maneira geral, constatou-se que o nível inferior de desempenho no teste TDE sob parâmetros de seriação escolar ocorreu independentemente de os alunos apresentarem indicação de deficiência e de prematuridade ou baixo peso ao nascimento. Contudo, esses fatores de risco adicionais acentuam a correlação entre o desempenho no teste de Integração Visuo-Motora do Beery VMI e o desempenho nas tarefas do TDE.

Este estudo busca contribuir para a ampliação do conhecimento no tocante à influência da habilidade de integração visuo-motora sobre o desempenho em tarefas escolares, abrindo perspectivas para discussões que venham ao encontro das necessidades educacionais inclusivas seja no âmbito das condutas preventivas de dificuldade ou distúrbio de aprendizagem seja no âmbito das condutas de intervenção.

Os resultados obtidos permitem ainda reiterar o que tem sido destacado na literatura quanto à oportunidade que a inclusão educacional oferece, no que tange à identificação de necessidades educacionais a partir de julgamentos com base no desempenho das tarefas realizadas e perante as demandas do ambiente e das tarefas em ambientes específicos.

Vale ressaltar as limitações do estudo no que tange ao número reduzido de crianças com os "fatores de risco adicionais" uma vez que a prematuridade, baixo peso e presença de deficiência são fatores que podem aumentar as chances de alteração no desenvolvimento neuromotor, cognitivo e acadêmico, no entanto cada um deles é bastante complexo podendo e devendo ser estudados de maneira individualizada e em uma amostra maior.

\section{Referências}

Ambrósio, M. F. S. (2011). A psicomotricidade e a alfabetização dos alunos no $2^{\circ}$ anos do ensino fundamental. Dissertação de mestrado, Universidade Estadual de Campinas, Campinas, SP, Brasil.

Beery, K. E., \& Beery, N. A. (2010). The Beery-Buktenica Developmental Test of Visual-Motor Integragion (6th ed.). Texas: Pearson. 
Braga, P. P., \& Sena, R. R. (2013). Avanços na atenção ao prematuro e a continuidade da assistência: reflexões sobre rede de cuidados. Revista de Enfermagem do Centro Oeste Mineiro, 3(3), 899-908.

Capellini, S. A., \& Souza, A. V. (2008). Avaliação da função motora fina, sensorial e perceptiva em escolares com dislexia do desenvolvimento. In A. L. Sennyey, F. C. Capovilla, \& J. M. Montiel (Orgs.), Transtornos de aprendizagem: Da avaliação à reabilitação (pp. 55-64). São Paulo, SP: Artes Médicas.

Carvalho, D. J., \& Magalhães, L. C. (2004). A relação entre o desenho da figura humana e a coordenação visuomotora em crianças pré-termo aos seis anos de idade. Revista de Terapia Ocupacional da Universidade de São Paulo, 15(3), 98-105.

Dias, R. S. (2004). Bases neuropsicológicas da aprendizagem: Atenção, percepção e memória. In: L. E. R. Valle (Org.), Temas multidisciplinares de neuropsicologia e aprendizagem. (pp. 393402). São Paulo, SP: Robe Editorial.

Formiga, C. K. M. R., \& Linhares, M. B. M. (2009). Avaliação do desenvolvimento inicial de crianças nascidas pré-termo. Revista da Escola de Enfermagem da USP, 43(2),472-480.

Gallahue, D. L., \& Ozmun, J. C. (2005). Compreendendo o desenvolvimento motor: Bebês, crianças, adolescentes e adultos (3a ed.). São Paulo: Phorte.

Gomes, J. A. D. G. (1998). Construção de coordenadas espaciais, psicomotricidade e desempenho escolar. Dissertação de mestrado, Universidade Estadual de Campinas, Campinas, SP, Brasil.

Haywood, K. M., \& Getchell, N. (2010). Desenvolvimento motor ao longo da vida (5a ed.). Porto Alegre: Artmed.

Kulp, M. T. (1999). Relationship between visual motor integration skill and academic performance in kindergarten through third grade. Optometry and Vision Science, 76(3), 159-163.

Lundy-Ekman, L. (2004). Neurociências: Fundamentos para a reabilitação. Rio de Janeiro: Elsevier.

Lustosa, N. P., Fiorentin, S., \& Rocha, D. L. S. (2004). Psicomotricidade e alfabetização. Anais do Congresso Nacional da Área de Educação, 2, \& EDUCERE, 4. Recuperado em 12 abril 2008, de http://www.pucpr.br/eventos/educere/educere2004/anaisEvent o/Documentos/ CI/ TC-CI0133.pdf

Magalhães, L. C., Catarina, P. W., Barbosa, V. M., Mancini, M. C., \& Paixão, M. L. (2003). Estudo comparativo sobre o desempenho perceptual e motor na idade escolar em crianças nascidas prétermo e a termo. Arquivos de Neuro-Psiquiatria, 61(2-A), 250255. 
Magalhães, L. C., Rezende F. C. A., Magalhães, C. M., \& Albuquerque, P. D. (2009). Análise comparativa da coordenação motora de crianças nascidas a termo e pré-termo aos 7 anos de idade. Revista Brasileira de Saúde Materno Infantil, 9(3), 293-300.

McHale, K., \& Cermark, S. A. (1992). Fine motor activities in elementary school: Preliminary findings and provisional implications for children with fine motor problems. The American Journal of Occupational Therapy, 46(10), 898-903.

Oliveira, R. T., Lopes, L. G., \& Magalhães, L. C. (2004). A relação entre integração visuomotora e escrita em crianças de 6 e 7 anos. Temas sobre desenvolvimento, 13(75), 5-13.

Pessoa, T. A. O., Martins, C. B. G., Lima, F. C. A., \& Gaíva, M. A. M. (2015). O crescimento e desenvolvimento frente à prematuridade e baixo peso ao nascer. Avances en Enfermería, 33(3), 401-411.

Pinelli Júnior, B. (1990). Adaptação do Teste do Desenvolvimento da Integração Viso-Motora (VMI) para uso no Brasil. Dissertação de mestrado, Universidade de Brasília, Brasília, DF, Brasil.

Riechi, T. I. J. (2008). Impacto do nascimento pré-termo e com baixo peso nas funções neuropsicológicas de escolares. Tese de doutorado, Universidade Estadual de Campinas, Campinas, SP, Brasil.

Riechi, T. I. J., Moura-Ribeiro, M. V. L., \& Ciasca, S. M. (2011). Impacto do nascimento pré-termo e com baixo peso na cognição, comportamento e aprendizagem de escolares. Revista Paulista de Pediatria, 29(4), 459-501.

Rodrigues, O. M. P. R., \& Bolsoni-Silva, A. T. (2011). Efeitos da prematuridade sobre o desenvolvimento de lactentes. Revista Brasileira de Crescimento e Desenvolvimento Humano, 21(1), 111-121.

Rosa Neto, F. (2002). Manual de avaliação motora. Porto Alegre: Artmed.

Sanghavi, R., \& Kelkar, R. (2005). Visual-motor integration and learning disabled children. The Indian Journal of Occupational Therapy, 37(2), 33-38.

Stein, L. M. (1994). TDE - Teste de Desempenho Escolar: Manual para aplicação e interpretação. São Paulo: Casa do psicólogo.

Suehiro, A. C. B., \& Santos, A. A. A. (2005). O Bender e as dificuldades de aprendizagem: Estudo de validade. Avaliação Psicológica, 4(1), 23-31.

Trevisan, J. G., Coppede, A. C., \& Capelini, S. A. (2008). Avaliação da função motora fina, sensorial e perceptiva em escolares com dificuldades de aprendizagem. Temas Sobre Desenvolvimento, 16(94), 183-187.

Zafani, M. D., \& Araújo, R. C. T. (2010). Programa de treinamento da percepção visual, coordenação motora e integração visuo- 
motora: Descrição do processo de elaboração. Anais do Congresso Brasileiro de Educação Especial, 4, \& Encontro da Associação de Pesquisadores em Educação Especial, 4, 47334743.

\section{Endereço para correspondência \\ Débora Morais Pereira}

Centro de Reabilitação Lucy Montoro

Unidade São José dos Campos - Setor Terapia Ocupacional

Rua Saigiro Nakamura, 600, Vila Industrial, CEP 12220-280, São José dos Campos - SP, Brasil

Endereço eletrônico: debora_terapi@yahoo.com.br

\section{Rita de Cássia Tibério Araújo}

Universidade Estadual Paulista Júlio de Mesquita Filho - UNESP

Campus Marília/SP

Departamento de Fisioterapia e Terapia Ocupacional, sala 35 (Bloco Educação)

Av. Hygino Muzzi Filho, 737, Mirante, CEP 17.535-000, Marília - SP, Brasil

Endereço eletrônico: ritac@marilia.unesp.br

\section{Ligia Maria Presumido Braccialli}

Universidade Estadual Paulista Júlio de Mesquita Filho - UNESP

Campus Marília/SP

Departamento de Fisioterapia e Terapia Ocupacional, sala 35 (Bloco Educação)

Av. Hygino Muzzi Filho, 737, Mirante, CEP 17.535-000, Marília - SP, Brasil

Endereço eletrônico: bracci@marilia.unesp.br

Recebido em: $12 / 11 / 2014$

Reformulado em: 15/03/2016

Aceito para publicação em: 31/03/2016

\section{Notas}

* Terapeuta Ocupacional do Centro de Reabilitação Lucy Montoro (Unidade São José dos Campos), Mestre em Educação, Especialista em Desenvolvimento Infantil.

** Docente do Programa de Pós-Graduação em Educação e do Departamento de Fisioterapia e Terapia Ocupacional da Universidade Estadual Paulista Júlio de Mesquita Filho - UNESP, campus de Marília/SP.

*** Docente do Programa de Pós-Graduação em Educação e do Departamento de Educação Especial da Universidade Estadual Paulista - UNESP - Marília, LivreDocente em Reabilitação Física, Doutora em Educação Física. 